\title{
Numerical Simulation of Particle Concentration in a Gas Cyclone Separator
}

\author{
Xue Xiaohu, Sun Guogang**, Wan Gujun and Shi Mingxian \\ (School of Chemical Science and Engineering, China University of Petroleum, Beijing, 102249, China)
}

\begin{abstract}
The particle concentration inside a cyclone separator at different operation parameters was simulated with the FLUENT software. The Advanced Reynolds Stress Model (ARSM) was used in gas phase turbulence modeling. Stochastic Particle Tracking Model (SPTM) and the Particle-Source-In-Cell (PSIC) method were adopted for particles computing. The interaction between particles and the gas phase was also taken into account. The numerical simulation results were in agreement with the experimental data. The simulation revealed that an unsteady spiral dust strand appeared near the cyclone wall and a non-axi-symmetrical dust ring appeared in the annular space and under the cover plate of the cyclone. There were two regions in the radial particle concentration distribution, in which particle concentration was low in the inner region $(r / R \leqslant 0.75)$ and increased greatly in the outer region $(r / R>0.75)$. Large particles generally had higher concentration in the near-wall region and small particles had higher concentration in the inner swirling flow region. The axial distribution of particle concentration in the inner swirling flow $(r / R \leqslant 0.3)$ region showed that there existed serious fine particle entrainment within the height of $0.5 \mathrm{D}$ above the dust discharge port and a short-cut flow at a distance of about $0.25 \mathrm{D}$ below the entrance of the vortex finder. The dimensionless concentration in the high-concentration region increased obviously in the upper part of the cyclone separation space when inlet particle loading was large. With increasing gas temperature, the particle separation ability of the cyclone was obviously weakened.
\end{abstract}

Key words: Cyclone separator, particle concentration distribution, simulation

\section{Introduction}

The turbulent flow field with strongly swirling flow in a cyclone is very complex. The particle flow and the interaction between particles and gas phase are even more complicated. Many investigations on gas and solids flows in a cyclone separator have been conducted over the years (Wu, et al.,1993; 1994a; 1994b; Wu and Shi, 2003; Cao, et al.,1994) and a number of empirical models for estimation of the performance of a cyclone were developed, but these models are not always suitable for different cyclones. The particle concentration in a cyclone is the basis for separation process study and new model development. However the experimental data of the particle concentration in the cyclones were rare and sparsely reported in literature due to the difficulties in measurement, especially for a cyclone at high temperature and high solids loading conditions. Along with the development of computer technology, computational fluid dynamics (CFD) codes have proved to be a useful tool for exploring the gas-solids flow in a cyclone, and may be a more generic way to know what is happening in the cyclones. The previous numerical research mainly aimed at the gas-phase flow field and the particle-trajectory (Lin and Wang, 1999; Wang, et al., 2006), and only a little attention was paid to the particle concentration in the cyclones (Xue, 2005). In order to gain some insights into the physical characteristics of the cyclone separation process, the effects of inlet particle loading, particle diameter and gas temperature on the particle concentration in a cyclone were numerical examined in present study.

\section{Model and method}

\subsection{Mathematical model}

For the unsteady and incompressible turbulent flow in cyclone, the Reynolds-stress Model (RSM) is frequently used for simulation ( $\mathrm{Lu}$ and Zhou, 2003). This model was also used in this study. Besides the time-averaged continuity equation and momentum equation, the transport equation of the stress components is expressed as follows (Hu, et al., 2005):

$$
\frac{\partial}{\partial t}\left(\rho \overline{u_{i} u_{j}}\right)+\frac{\partial}{\partial x_{k}}\left(\rho U_{k} \overline{u_{i} u_{j}}\right)=D_{i j}+P_{i j}+\Pi_{i j}-\varepsilon_{i j}
$$

where, the left side of the equation is the local time derivative and convection term, respectively. $D_{i j}, P_{i j}, \Pi_{i j}$ and $\varepsilon_{i j}$ are stress diffusion, stress production, pressure-strain and stress dissipation rate terms, respectively.

The closed model of pressure-strain term, $\Pi_{i j}$, concerns the reasonableness of the prediction of Reynolds stress. After selecting from several available linear and nonlinear models, such as IPM (Gibson and

\footnotetext{
* Supported by the National Key Project of Basic Research of the Ministry for Science and Technology of P. R. China (Grant No. 2005CB22120103)

** Corresponding author: Prof. SUN Guogang. E-Mail: ggsun@163bj.com
} 
Launder, 1978), IPCM (Fu, et al., 1987), GL (Launder, 1989a), SSG (Launder, 1989b), FLT (Speziale, et al., 1991), the Isotropization of Production and Convection Model (IPCM) and the wall model were used. In order to predict the Reynolds stress better, an improved model for strongly swirling flow (Hu, et al., 2004) was used, in which the model constant was optimized in consideration of the strongly swirling flow in the cyclone.

The stochastic Particle Tracking Model describes the particle motions by tracking particle trajectories in a Lagrangian frame of reference. It is suitable for numerical simulation of dilute gas-particle two-phase flows where the volume fraction of the particle phase is small, which is similar to the particle concentrations in most parts of a cyclone. Therefore, in this paper, the Stochastic Particle Tracking Model was selected to simulate the particle trajectory, and the Particle-Sourcein-Cell (PSIC) method (Cen and Fan, 1990) is used to calculate the particle concentration. Generally, the particle concentration, $C_{\mathrm{i}}$, in the cyclone entrance is less than $10 \mathrm{~kg} / \mathrm{m}^{3}\left(C_{\mathrm{i}} \leqslant 10 \mathrm{~kg} / \mathrm{m}^{3}\right)$. The particle volume fraction, $\varepsilon=C_{\mathrm{i}} / \rho_{\mathrm{b}}=0.37 \%-0.67 \%<1 \%$. Since the particle concentration in most parts of a cyclone $(\varepsilon<<1 \%)$ belonged to the dilute phase except in the wall region, the particle-particle interaction could be neglected. Only the gas-particle interaction and the dispersion of particles due to turbulence were taken into account. Therefore, the particle motion equations in a Lagrangian frame of reference could be expressed as:

$$
\begin{aligned}
& \text { Axial } \frac{\mathrm{d} u_{\mathrm{p}}}{\mathrm{d} t}=\frac{1}{\tau}\left(u_{\mathrm{g}}+u_{\mathrm{g}}-u_{\mathrm{p}}\right)-g \\
& \text { Radial } \frac{\mathrm{d} v_{\mathrm{p}}}{\mathrm{d} t}=\frac{1}{\tau}\left(v_{\mathrm{g}}+v_{\mathrm{g}}-v_{\mathrm{p}}\right)+\frac{w_{\mathrm{p}}^{2}}{r_{0}} \\
& \text { Tangential } \frac{\mathrm{d} w_{\mathrm{p}}}{\mathrm{d} t}=\frac{1}{\tau}\left(w_{\mathrm{g}}+w_{\mathrm{g}}-w_{\mathrm{p}}\right)-\frac{u_{\mathrm{p}} w_{\mathrm{p}}}{r_{0}}
\end{aligned}
$$

where, $\mathrm{p}, \mathrm{g}$ stands for the particles and gas phase, respectively. $r_{0}$ is the radial position of particles. $\tau$ stands for the relaxation time of particles., $u_{g}^{\prime}, v_{g}^{\prime}$ and $w_{g}^{\prime}$ are the fluctuation velocity components.

$$
\tau=\frac{\rho_{\mathrm{p}} d_{\mathrm{p}}^{2}}{18 \mu} \cdot \frac{24}{C_{\mathrm{D}} \operatorname{Re}_{\mathrm{p}}}
$$

where, $\rho_{\mathrm{p}}$ is particle density. $d_{\mathrm{p}}$ is particle diameter. $\mu$ is the molecular viscosity of the gas. The drag coefficient,

$C_{\mathrm{D}}$, can be obtained from $C_{\mathrm{D}}=a_{1}+\frac{a_{2}}{\mathrm{Re}_{\mathrm{p}}}+\frac{a_{3}}{\mathrm{Re}_{\mathrm{p}}^{2}}$, where $a_{1}, \quad a_{2}$ and $a_{3}$ are constants for smooth spherical particles over several ranges of $R$ e given by Morsi and Alexander (Morsi and Alexander, 1972). R $\mathrm{e}_{\mathrm{p}}$ is the particle Reynolds number.

From gas momentum equations, the influence of temperature on the gas flow field is shown by their influence on gas viscosity and density. Hence, the gas viscosity and density were expressed as the functions of temperature in the numerical simulation. The relationship between gas viscosity and temperature is as follows:

$$
\mu_{T}=\mu_{0}\left(\frac{T}{T_{0}}\right)^{m}
$$

where $m$ is an experiential exponential, which is 0.683 for air.

According to the gas law, gas density is expressed by

$$
\rho_{T}=\rho_{0}\left(\frac{T_{0}}{T}\right)\left(\frac{P}{P_{0}}\right)
$$

\subsection{Boundary conditions and grids}

Fig. 1 shows the geometry of the simulated cyclone. The gas inlet dimension is $176 \mathrm{~mm} \times 84 \mathrm{~mm}$. The body inner diameter is $300 \mathrm{~mm}$. Structure grid was used for calculation, and the grid of the cyclone separator is shown in Fig. 2. The number of grid nodes is 978,653 .

The cyclone inlet air velocity was $20 \mathrm{~m} / \mathrm{s}$. The initial position of particles was on the cross-section of the inlet of cyclone, with the distribution of all the particles being uniform. Particles were injected from every grid center with the same velocity of the inlet gas. The calculated particle diameter (or diameter distribution), particle concentration and the number of particles' random tracks were all defined at the cyclone inlet cross-section

It was assumed that there was no mass change and no heat transfer during the process of particles motion. The 325 mesh talcum powder with a density of about $2,700 \mathrm{~kg} / \mathrm{m}^{3}$ was selected as the simulation particles, and the diameter distribution of which is shown in Table 1 .

Table 1 Diameter distribution of 325 mesh talcum powder

\begin{tabular}{lllllllll}
\hline$d_{\mathrm{p}}, \mu \mathrm{m}$ & 24 & 19 & 15 & 12 & 10 & 7 & 4 & 1 \\
\hline$M_{\mathrm{D}}, \%$ & 10 & 25 & 31 & 39 & 46 & 58 & 74 & 93 \\
\hline
\end{tabular}

Assuming that the diameter distribution of particles satisfies the Rossin-Rammler distribution, the following equation for particle size distribution can be obtained by regression of the data in Table 1: 


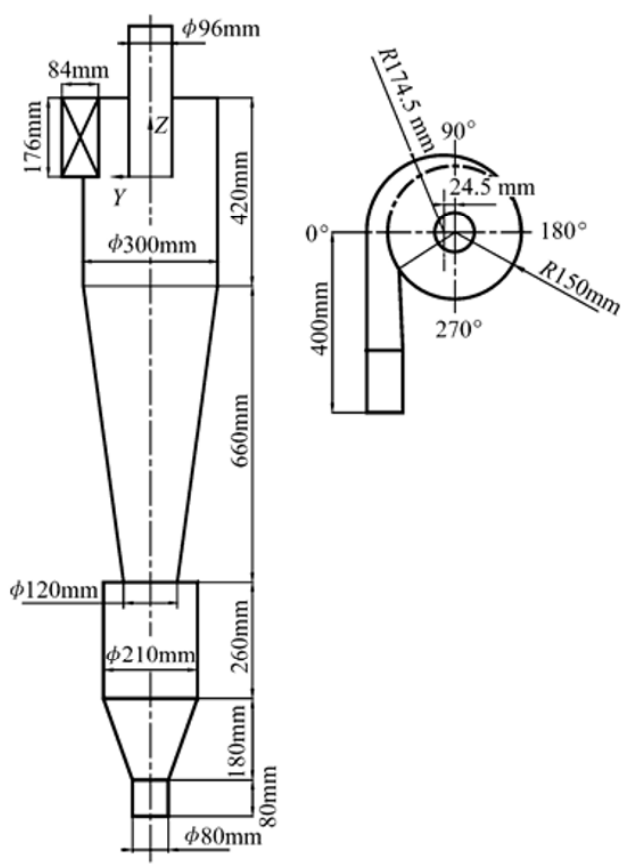

Fig. 1 Schematic diagram of the cyclone

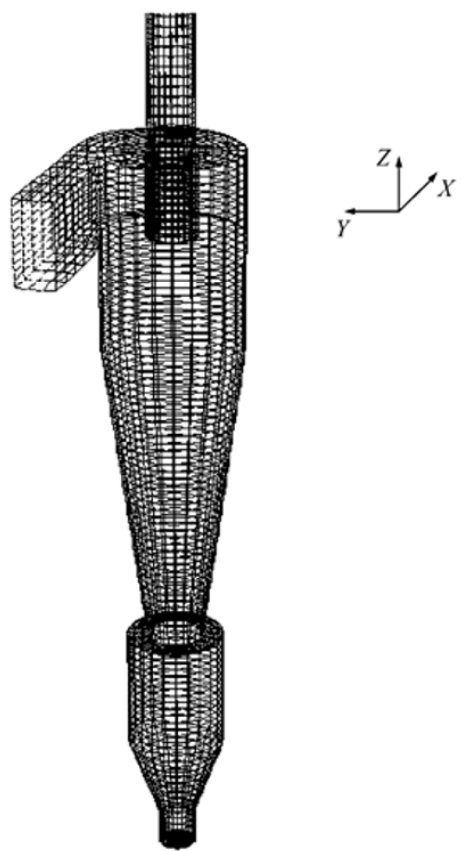

Fig. 2 The grid of cyclone separator

$$
M_{D}=\exp \left[-\left(d_{\mathrm{p}} / 12.74\right)^{1.858}\right]
$$

The exit boundary condition of the continuous phase was considered as a fully developed tube flow, assuming all the flow variables with a zero axial gradient on the exit cross-section, i.e. $\frac{\partial \varphi}{\partial Z}=0$. In order to ensure the flow to be fully developed, the cyclone exit tube was prolonged. The exit boundary condition of particles was assumed to be that when it ran into the exit the particle could escape from the cyclone. A no-slip boundary condition was used at the wall in calculation of the gas-phase flow field. Standard wall functions were used to model the flow near the wall region. The particle-wall boundary condition was considered as damped elastic collision. The coefficient of restitution was set as 1.0-0.90 for the top annular space of cyclone, 0.90-0.60 for the middle separation space and 0.60-0.05 for the dust hopper.

The QUICK discretization and SIMPLE-C algorithm were used to calculate the cyclone flow field, associated with unsteady particle trajectory tracking calculations. The gas flow field and particle trajectory were calculated alternately until a converged solution was achieved.

\section{Results and discussion}

\subsection{Test of the simulation results}

To judge the creditability of the simulation results, the numerically calculated result was compared with the experimental data in literature (Wu, et al., 1994a). Fig. 3 shows the measured and the calculated radial particle concentration distribution on the cross section at a distance of $600 \mathrm{~mm}(Z=-600 \mathrm{~mm})$ below the vortex finder entrance. Fig. 4 shows the comparison of the measured and the calculated results of the axial particle concentration distribution at the radial position of $r / R=0.3$. The comparisons indicted that the calculated result of the particle concentration distribution was satisfactory.

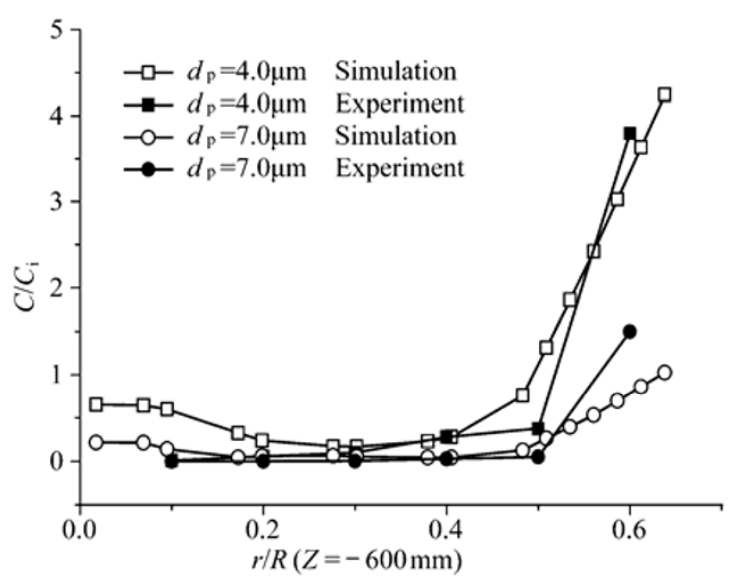

Fig. 3 Simulated and measured radial particle concentration distribution in separation space $(Z=-600 \mathrm{~mm})$ 


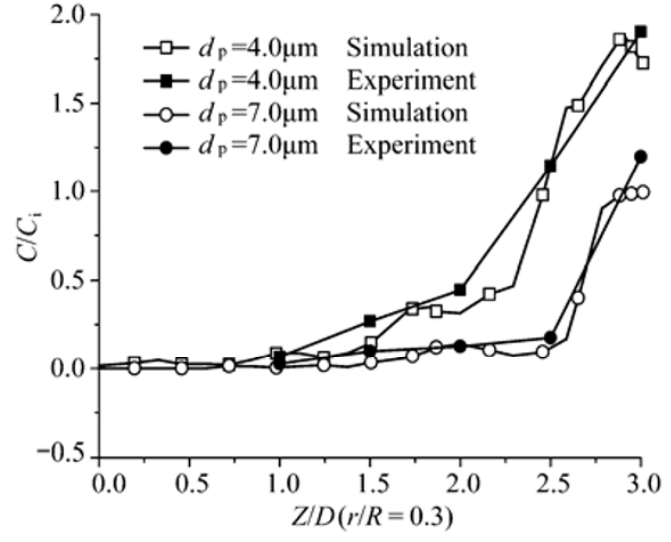

Fig. 4 Simulated and measured axial particle concentration distribution $(r / R=0.3)$

\subsection{Basic characteristics of particle concentration in a cyclone}

Figs. 5-6 show the simulated particle concentration on the wall at two moments ( $t=2.7$ and $3 \mathrm{~s})$. Figs. 7-9 display the particle concentrations in the annular space, separation space and dust hopper of the cyclone.

Figs. 5-6 show the spiral dust strand near the cyclone wall and its unsteady characteristics, and space domains, which undulated with a frequency. But its dimensionless concentration did not change obviously, ranging from 15 to 30. Figs. 7-9 show that there existed dust rings in the upper part of the annular space and the dust hopper, and the particle concentration in the dust rings was not uniform nor axi-symmetric distribution. There existed a short-cut flow at a distance of about $0.25 D$ below the entrance of the vortex finder. Fig. 10 shows that the particles were entrained seriously from the dust hopper in a range of about $0.5 \mathrm{D}$ above the dust discharge port, and the secondary separation of particles due to the strongly swirling flow also occurred obviously in this region. The radial particle distribution in the separation space could be divided into the low-concentration region in the center and the high-concentration region near the wall. In most parts of the annular space and of the separation space, the low-concentration region was approximately $0-0.75 R$ and the high-concentration region was about $(0.75-1.0) R$. The particles concentration increased sharply at a distance of about $0.85 R$ in the annular space at $0^{\circ}$ orientation. In a range of $0.5 \mathrm{D}$ above the dust discharge port, the particle concentration was higher in the inner swirling flow region. In the dust hopper, there was a high-concentration region near the wall and under the top plate of hopper, but no remarkable difference in particle concentration in the other parts of the hopper.
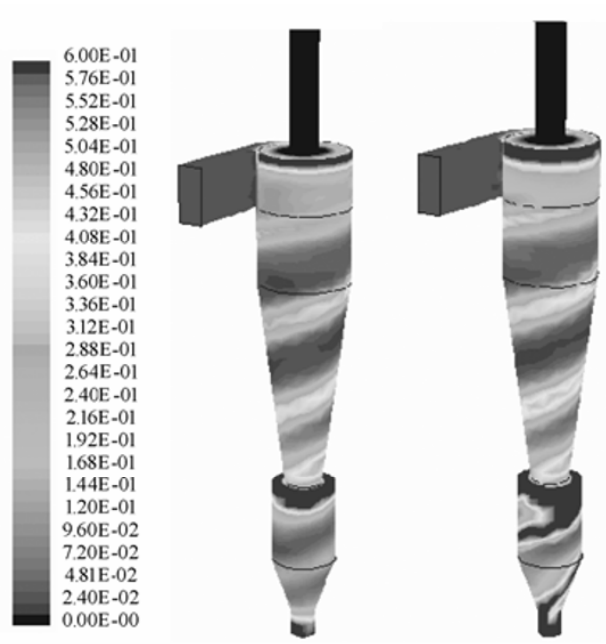

(a) $t=2.7 \mathrm{~s}$

(b) $t=3 \mathrm{~s}$

Fig. 5 Particle concentration on the wall at two moments

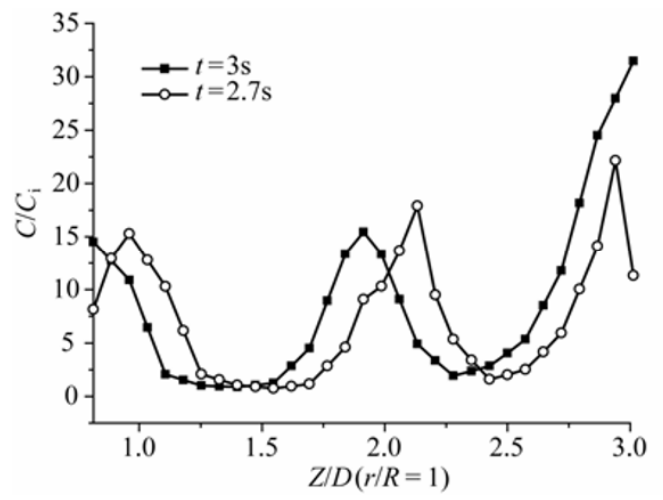

Fig. 6 Axial particle concentration distribution on the cyclone wall at two moments

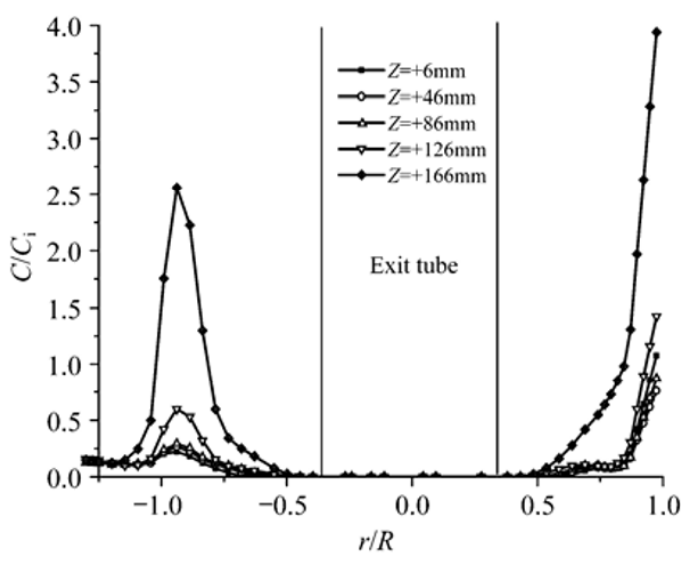

Fig. 7 Radial particle concentration distribution in the annular space of the cyclone 


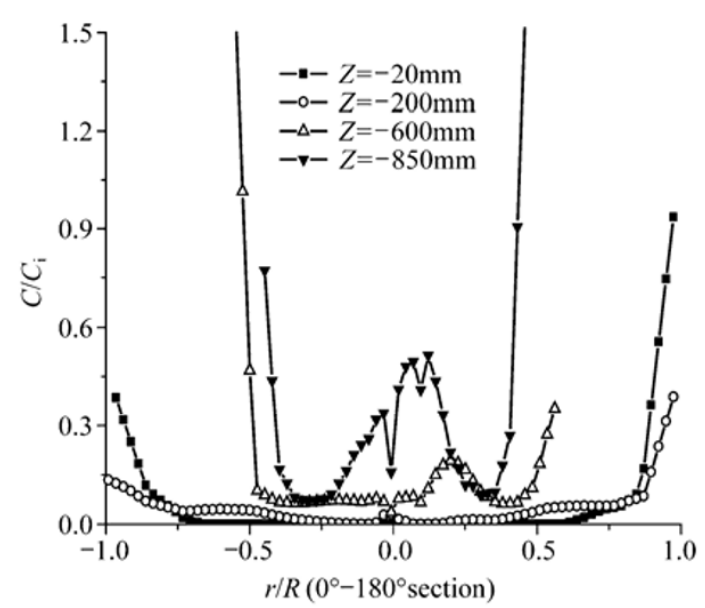

Fig. 8 Radial particle concentration distribution in the separation space of the cyclone

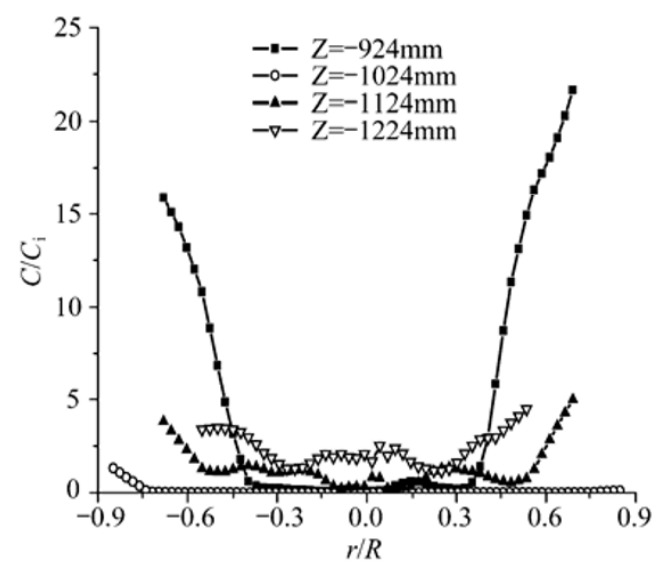

Fig. 9 Radial particle concentration distribution in the dust hopper

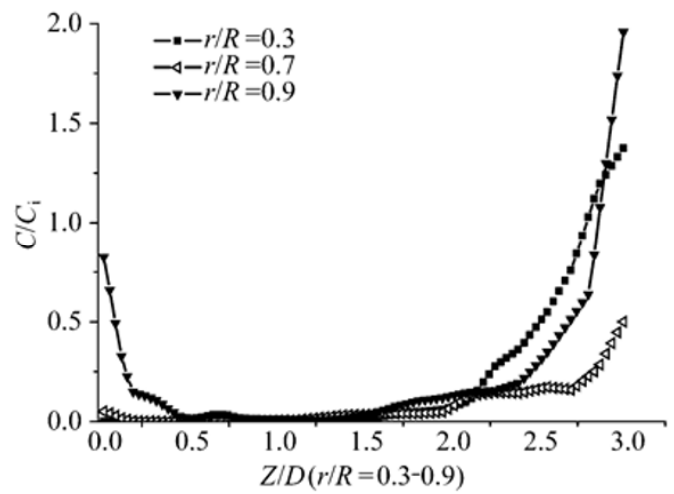

Fig. 10 Axial particle concentration distribution in the cyclone

\subsection{Distribution of particles size}

Fig. 11 shows the radial particles concentration distribution of several particle sizes in the separation space of the cyclone. Fig. 12 is axial particles concentration distribution of different particle diameters in the inner swirling flow region $(r / R=0.3)$ of the cyclone.
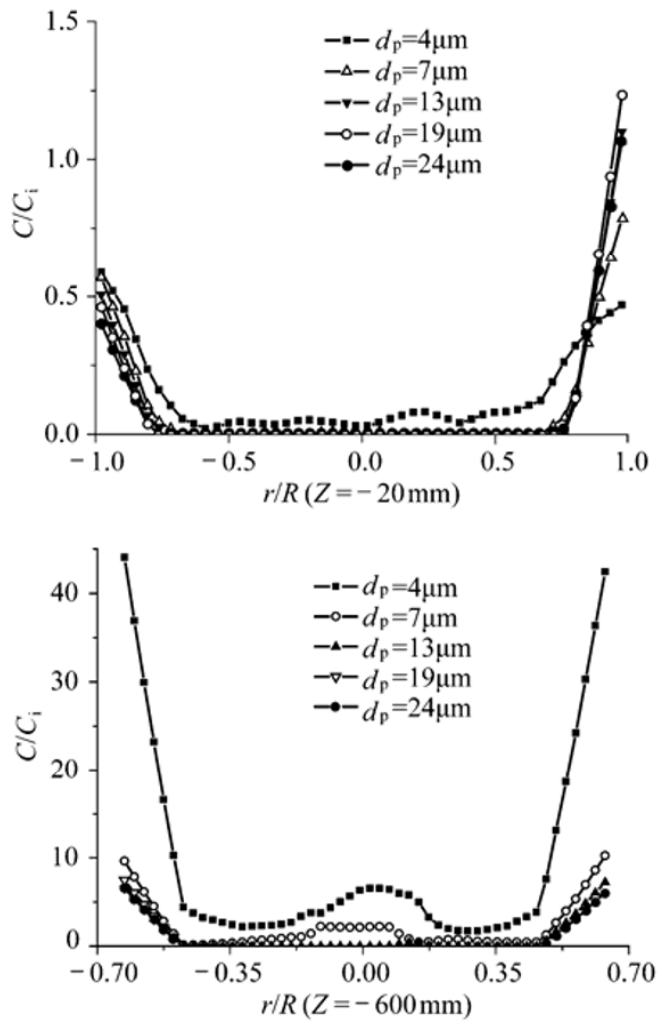

Fig. 11 Radial particle concentration distribution of different particle sizes at the distance of $20 \mathrm{~mm}$ and $600 \mathrm{~mm}$ under vortex finder

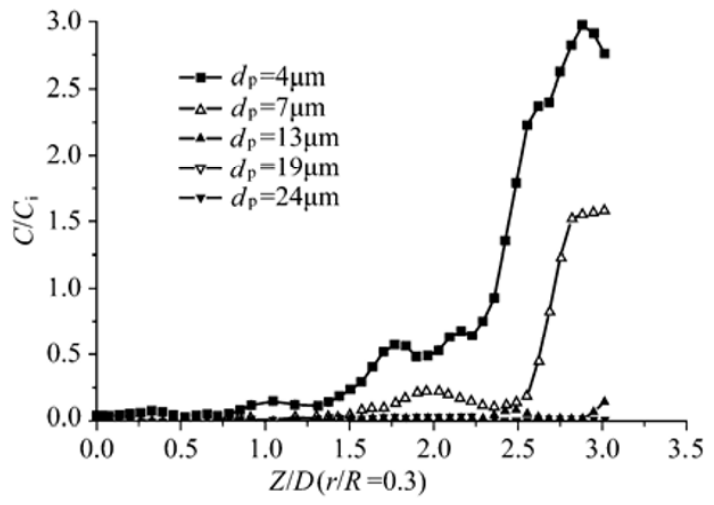

Fig. 12 Axial particle concentration distribution of different particle sizes in inner swirling flow region $(r / R=0.3)$

It is seen from Fig. 11 that in the upper part of the cyclone, the dimensionless particle concentration of different particle diameters did not change greatly in the high-concentration region near the wall. While in the lower part of the cyclone, smaller particles always had higher concentration. The larger the particles (e.g. $\left.d_{\mathrm{p}}>10 \mu \mathrm{m}\right)$, the bigger the centrifugal force acting on the particles. Thereby the larger particle moved faster towards the wall and resulted in a higher concentration near the wall region. While for the small particles, because of small centrifugal force and the effect of turbulent dispersion, the separation was insufficient, 
resulting in a high-concentration in the region under the vortex finder and in the inner swirling flow region of the cyclone separation space. Meanwhile, the simulation revealed that most particles entrained from the dust discharge port were of less than $10 \mu \mathrm{m}$ in diameter. Particles larger than $6 \mu \mathrm{m}$ in diameter could be separated again by the secondary separation in the zone above the dust discharge port. However particles less than $5 \mu \mathrm{m}$ were hard to be separated in the secondary separation zone. This is one reason of low efficiency of separation for small particles.

\subsection{Effect of inlet particle loading}

Fig. 13 and Fig.14 show the particle concentration distribution at different cyclone inlet particles loadings. When inlet particle loading increased (e.g. $C_{\mathrm{i}}>2 \mathrm{~kg} / \mathrm{m}^{3}$ ), the center low-concentration region decreased to about $r \leqslant 0.5 \mathrm{R}$ and the high-concentration region increased to $0.5 R<r \leqslant R$ (see Fig. 13). The dimensionless concentration in the high-concentration region increased obviously in the upper part of the cyclone separation space when inlet particle loading was large. But in the cyclone conical part, the influence of inlet particles loading on particle concentration was not obvious. It indicated that when inlet particle loading was high, separation was mainly carried out in the upper separation space. Thereby, in order to improve the separation efficiency, the cyclone body should be prolonged appropriately. The short-cut flow region below the entrance of vortex finder still maintained in the range of $0.25 D$, but the dimensionless particle concentration would increase obviously. The range of the particle entrainment region above the dust discharge port would reduce to less than $0.5 D$ with particle concentration decreasing significantly. Thus, increasing the cyclone separation space height and reducing the exit short-cut flow are of significance for a better separation.

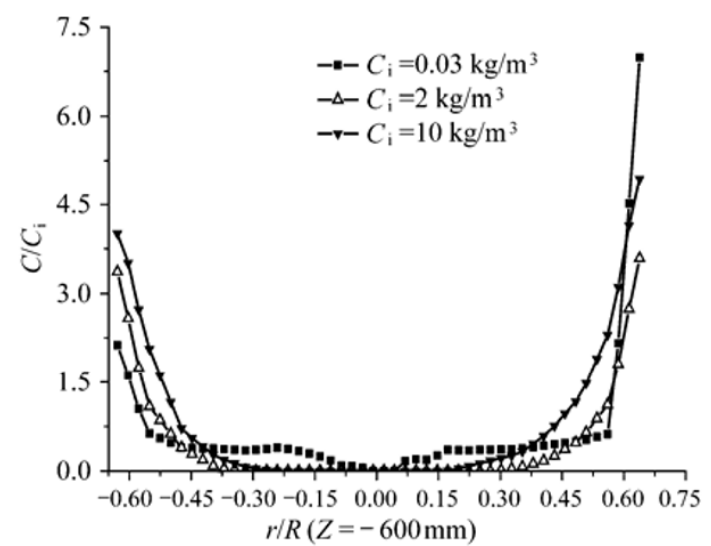

Fig. 13 Radial particle concentration distribution at a distance of $600 \mathrm{~mm}$ under the vortex finder

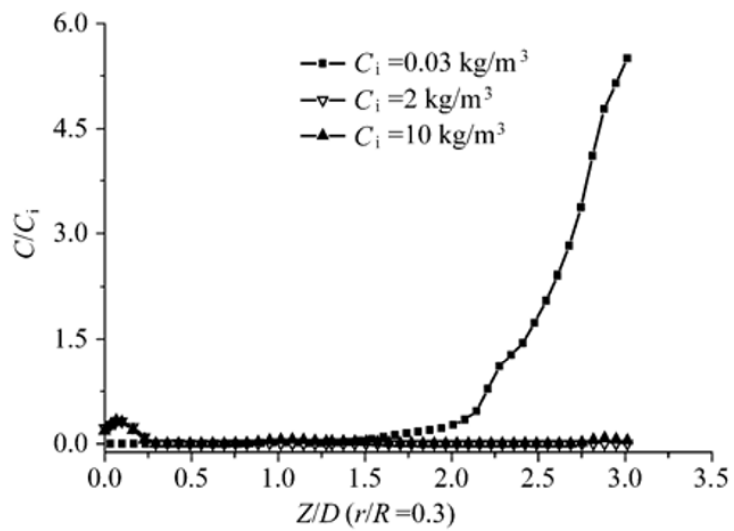

Fig. 14 Axial particle concentration distribution in the inner swirling flow region $(r / R=0.3)$

\subsection{Effect of gas temperature on particle concentration}

Figs. 15-18 display the particle concentration distributions at different gas temperatures. With increasing gas temperature, the particle separation ability of the cyclone was obviously weakened. This is mainly because the viscosity of the gas increased with increasing temperature and the drag force acting on the particle also increased. Meanwhile, when the temperature rose, the turbulent fluctuation of the gas obviously was strengthened too, causing increased turbulent dispersion of the fine particles. So, the separation possibility of the particles was significantly reduced. When temperature rose, the dimensionless particle concentration in the high-concentration region did not change obviously in the upper area of the cyclone separation space. However it increased significantly in the near wall area of the conical part of the cyclone separation space. It indicated that at a high temperature, the particle separation process was not finished in the annular space and the upper part of the separation space of a cyclone and continued in its lower conical part of the cyclone separation space. Thereby, present simulations suggested that a cyclone for high-temperature operation should have a higher ratio of height to body radius. From the calculations, it was also seen that the upper short-cut flow region and the bottom particle entrainment region were obviously increased at a high temperature $(T \geqslant 1273 \mathrm{~K})$. And the two regions even overlapped, resulting in sharply worsened separation performance.

\section{Conclusion}

1) The particle concentration distribution in a cyclone was numerically investigated with the Particle-Source-In-Cell (PSIC) method and incorporation of gas-particle interaction. The calculation results were in good agreement with the measured data in literature (Wu, et al., 1994a). 


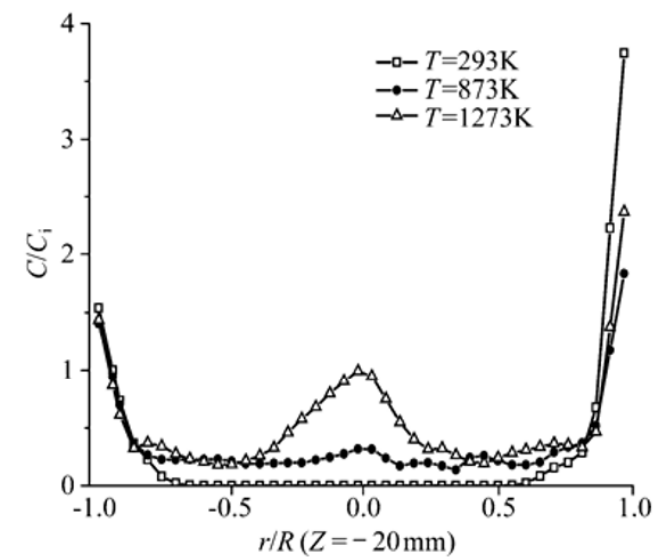

Fig. 15 Radial particle concentrations at a distance of $20 \mathrm{~mm}$ below the vortex finder

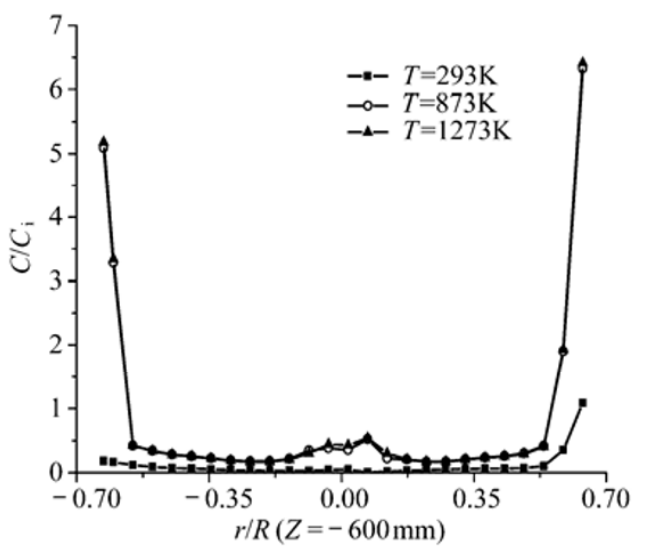

Fig. 16 Radial particle concentrations at a distance of $600 \mathrm{~mm}$ below the vortex finder

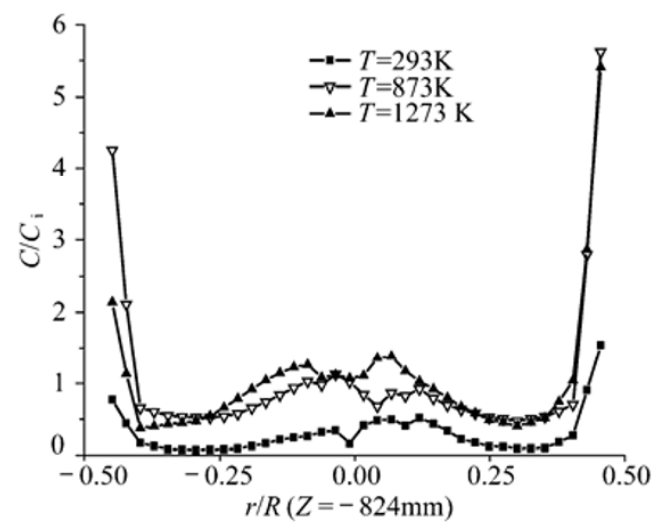

Fig. 17 Radial particle concentrations at a distance of $824 \mathrm{~mm}$ below the vortex finder

2) The radial distribution of particle concentration was divided into the low-concentration region in the center and the high-concentration region near the wall in the cyclone separation space. The particle concentration near the cyclone wall was simulated as an unsteady spiral dust strand. High-concentration dust rings existed in the cyclone annular space and under the cover plate of the dust hopper, and the concentrations in the dust rings were not uniform nor axi-symmetric. A short-cut flow appeared below the entrance of the vortex finder. The particles were entrained seriously from the dust hopper, but the secondary separation of particles due to the strongly swirling flow was also obvious in the conical part.

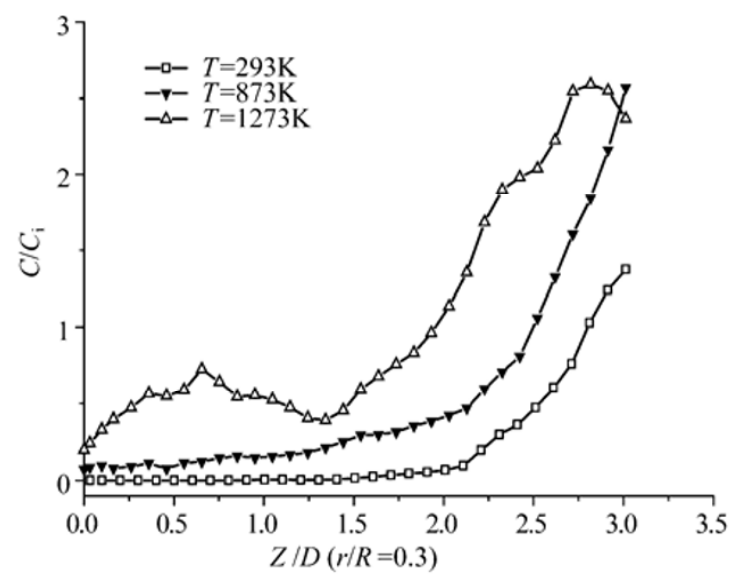

Fig. 18 Axial particle concentrations in the inner swirling flow region $(r / R=0.3)$

3) As the inlet particle loading increased, the radial low-concentration region decreased and the high-concentration region increased. The dimensionless concentration in the short-cut flow region increased obviously with increasing inlet solids loading.

4) The separation efficiency of cyclone generally decreased with increasing gas temperature. The upper short-cut flow region and the bottom particle entrainment region would link up together and even overlap at a high temperature.

\section{Nomenclatures}

$C$ - Particles concentration, $\mathrm{kg} / \mathrm{m}^{3}$

$C_{\mathrm{i}}$-Inlet particles concentration, $\mathrm{kg} / \mathrm{m}^{3}$

$D$ - Cyclone separator diameter, $\mathrm{mm}$

$d_{\mathrm{p}}$-Particle diameter, $\mathrm{mm}$

$M_{\mathrm{D}}$-Particle cumulative rate

$P$ - Pressure, $\mathrm{Pa}$

$P_{0}$ - Normal conditions pressure, $\mathrm{Pa}$

$r$ - Cyclone separator radial position, $\mathrm{mm}$

$R$ - Cyclone separator radius, $\mathrm{mm}$

$T$ - Temperature, $\mathrm{K}$

$T_{0}$ - Normal conditions temperature, $\mathrm{K}$

$Y$ - Radial coordinate, $\mathrm{mm}$

$Z$ - Axial coordinate, $\mathrm{mm}$

$\mu_{0}$ - Normal conditions gas viscosity, $\mathrm{Pa} \cdot \mathrm{s}$

$\mu_{T}$ - Gas viscosity at high temperature, $\mathrm{Pa} \cdot \mathrm{s}$

$\rho_{0}$-Normal conditions gas density, $\mathrm{kg} / \mathrm{m}^{3}$

$\rho_{T}$ - Gas density at high temperature, $\mathrm{kg} / \mathrm{m}^{3}$ 


\section{References}

Cao Y., Sun T. and Shi M. X. (1994) Study on fine particle entrainment from dust hopper of the cyclone separator, Proc. 2nd China-Japan Joint International Conference on Filtration \& Separation, 281-284

Cen K. F. and Fan. J. R. (1990) Engineering gas-solid multiphase flow theory and calculation. Hangzhou: Zhejiang University Press (in Chinese)

Fu S., Launder B. E. and Leschziner M. A. (1987) Modeling strongly swirling recirculating jet flow with Reynolds-stress transport closures. In Sixth Symposium on Turbulent Shear Flows, Toulouse, France.

Gibson M. M. and Launder B. E. (1978) Ground Effects on pressure fluctuations in the atmospheric boundary layer. $J$. Fluid Mech., 86, 491-511

Hu L. Y., Shi M. X., Zhou L. X. and Zhang J. (2004) Numerical simulation of 3-D strongly swirling turbulent flow in a cyclone separator. Journal of Hsinghua University, 44(11), 1501-1504. (in Chinese)

Hu L. Y., Zhou L. X., Shi M. X., et al. (2005) A modified pressure-strain model for simulating strongly swirling flows in a cyclone separator. Engineering Mechanics, 22(5), 83-88

Launder B. E. (1989a) Second-moment closure and its use in modeling turbulent industrial flows. International Journal for Numerical Methods in Fluids, 9, 963-985

Launder B. E. (1989b) Second-moment closure: Present and future? Inter. J. Heat Fluid Flow, 10(4), 282-300

Lin W. and Wang N. N. (1999) Numerical simulation of three dimensional and two phase flow in a cyclone separator, Power Engineering, 19(1),72-76. (in Chinese)

Lu Y. J. and Zhou L. X. (2003) Numerical simulation of fluid flow and oil-water separation in hydrocyclones. Chinese Journal of Chemical Engineering, 11(1), 97-101

Morsi S. A. and Alexander A. J. (1972) An investigation of particle trajectories in two-phase flow systems. J. Fluid Mech., 55(2), 193-208.

Speziale C. G., Sarkar S. and Gatski T. B. (1991) Modelling the pressure-strain correlation of turbulence: an invariant dynamical systems approach. J. Fluid Mech., 227, 245-272
Wang B., Xu D. L., Xiao G. X., et al. (2006) Numerical study of gas-solid flow in a cyclone separator. Applied Mathematical Modelling, 30, 1326-1342

Wu X. L., Huang X. D. and Shi M. X. (1993) Experimental study on particle concentration distribution in cyclone separator. $J$. of University of Petroleum, 17(4), 54-59 (in Chinese)

Wu X. L., Cao, Y. and Shi M. X.. (1994a) Experimental study on particle concentration distribution in PV cyclone separator, Proc. Chinese Chemical Engineering Annual Meeting 78-81 (in Chinese)

Wu X. L., Sun T. and Shi M. X. (1994b) The influence of configuration and operation parameters on particle concentration distribution in cyclone separator, The 2nd China-Japan Joint International Conference on Filtration \& Separation, 285-289

Wu X. L. and Shi M. X. (2003) Visualization of the processing vortex core in a cyclone separator by PIV. Chinese Journal of Chemical Engineering, 11(6), 633-637

Xue X. H. (2005) Numerical investigation on the gas-particle two-phase flow behavior in the full space of the cyclone separator, Ph.D. Thesis, China University of Petroleum, Beijing (in Chinese)

\section{About the first author}

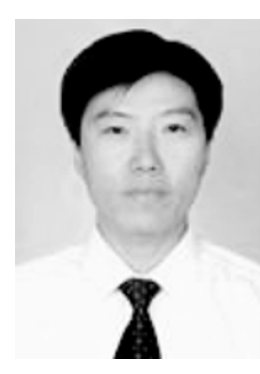

Xue Xiaohu was born in 1965. He received his $\mathrm{PhD}$ from the China University of Petroleum (Beijing) in 2005. As a Professorial Senior Engineer, he now works at China National Petroleum Corporation Services \& Engineering Ltd, with his interests in the field of numerical simulation of gas-particle two-phase flow. E-mail: xuexh@cptdc.cnpc.com.cn

(Received January 22, 2007) (Edited by Zhu Xiuqin) 